\title{
A Case Report of Rickettsial Meningitis
}

\author{
Sadia Islam ${ }^{1}$, Syed Wahidur Rahman ${ }^{2}$, Tasrina Shamnaz Samdani ${ }^{3}$
}

\begin{abstract}
Rickettsial diseases are a group of infections caused by the obligate intracellular bacteria Rickettsia. Rickettsial infections are common in southern Europe. The disease is usually characterized by the classical triad of fever, eschar and rash. Complications including neurological involvement are rarely described. We report an unusual case of meningitis in a 55 years old man presenting with high grade fever for 8 days associated with persistent headache. The patient was suspected to have enteric fever and treated with injection ceftriaxone. Forty-eight hours after admission, the presence of continued high grade fever, conjunctival congestion, headache, cough, low platelet and increased $W B C$ count, and elevated transaminase raised the possibility of a different aetiology. Finally, Rickettsial meningitis was confirmed due to history of field visit, presence of eschar, neck rigidity, raised titre of WF OX-K antigen and the cerebrospinal fluid analysis revealed increased cellularity, hypoglycorrhachia, and hyper proteinorrhachia $(106 \mathrm{mg} / \mathrm{dL})$. Patient was treated with capsule doxycycline and other supportive therapy and became afebrile within 48 hours. This uncommon clinical scenario should be taken into account in the management of patients with high grade fever on admission.
\end{abstract}

Key words: Rickettsia; rickettsial meningitis; eschar.

Delta Med Col J. Jul 2019;7(2):100-103

\section{Introduction}

Rickettsia may be responsible for certain febrile illness of unknown origin. It is often misdiagnosed and treated as other febrile illness because of its similarity of symptoms and lack of confirmation by laboratory investigations. When untreated, Rickettsial diseases can be fatal sometime. Co-infection of typhoid and Rickettsial fever can be overlooked if not suspected clinically. High grade continued fever with variable associated symptoms, such as malaise, headache, and myalgia, are usually present in both typhoid and Rickettsial fever. Therefore, clinical suspicion is of paramount importance in the diagnosis of both typhoid and Rickettsial fever. Confirmation of the diagnosis is important as the treatment is different but may not always be possible. In most cases of Rickettsial fever, treatment is given based on clinical suspicion due to presence of eschar or a positive Weil-Felix test. In clinically-suspected cases of typhus fever, a rising titre of OX-K

1. Associate Professor (CC), Dept. of Medicine, Delta Medical College, Dhaka, Bangladesh.

2. Professor, Dept. of Medicine, Delta Medical College, Dhaka, Bangladesh.

3. Assistant Professor, Dept. of Medicine, Enam Medical College, Savar, Dhaka, Bangladesh.

Correspondence: Dr. Sadia Islam. e-mail: Sadia_8899@yahoo.com 
antigens supports the diagnosis but confirmation of the diagnosis may be difficult. Isolation of Salmonella typhi from blood confirms the diagnosis of typhoid fever. Here, a case of Rickettsial meningitis admitted to the Delta Medical College and Hospital, having an eschar and highly rising titre of OX-K antigen in Weil Felix reaction is presented.

\section{Case Report}

In March 2018, a previously-healthy 55-year old non-smoker, non-alcoholic, married, fisherman hailing from Cumilla was admitted with complaints of high grade continued fever for 10 days associated with vomiting, abdominal discomfort, headache, body ache, and cough. No significant past illness was reported. He had no history of visiting in malaria endemic zone in recent past. On admission, the patient was conscious, oriented but dehydrated with high fever $\left(105^{\circ} \mathrm{F}\right)$. His radial pulse was $120 \mathrm{bpm}$, regular and good in volume; respiration rate was 20 per minute; and BP was 120/80 $\mathrm{mmHg}$. On examination, there were no sign of anaemia and jaundice. There was no skin rash, lymphadenopathy or neck rigidity. On systemic examination his breath sounds were vesicular, with no added sound abdomen was soft and non-tender, no hepatosplenomegaly and bowel sound was present. First and second heart sounds were normal without any murmur. Neurological and musculoskeletal system examination revealed normal findings. Relevant investigations were sent and intravenous (IV) ceftriaxone 2 gm 12 hourly was started. Laboratory investigations showed that total WBC was $16000 /$ cumm with polymorphs $56 \%$, lymphocyte $38 \%$, and monocyte $6 \%$ and platelet count was $95 \times 10^{3} / \mu \mathrm{L}$ with raised transaminases. His renal function tests, chest X-ray, urine routine examination, USG of whole abdomen were normal.

After 24 hours, the patient was found to be toxic, hyperthermic and developed conjunctival congestion. After two days, the patient was disoriented, and neck rigidity was present.
To further investigate, CSF study was done and it reavealed increased cellularity, hypoglycorrhachia, and hyper proteinorrhachia (106 mg/dL). Gram stain (no organism found) and ZN stain (AFB No found), GeneXpert for detection of MTB were negative. PBF for MP was absent, ICT for Malaria and Kala-Azar were negative.

On the 5th day of admission, the patient suddenly developed hypotension not responding to IV fluid challenge and on re-examination of the patient there was eschar on back of the chest (left side) and regional lymphadenopathy along with fever $105^{\circ} \mathrm{F}$ and blood pressure $70 / 50 \mathrm{~mm}$ of $\mathrm{Hg}$. Blood culture revealed no growth. Widal titers were not rising. Reconsidering all the investigations febrile antigen was done where Weil-Felix titres were: $\mathrm{OX} 2=1: 80, \mathrm{OX} 19=1: 80$, and $\mathrm{OXK}=1: 640$. Then the patient was treated with oral doxycycline 100 mg 12 hourly and other supportive therapy. After getting 4 doses of doxycycline patient became afebrile, clinically improved, there was no neck rigidity and patient regained consciousness. Finally, the patient was diagnosed with Rickettsial meningitis.

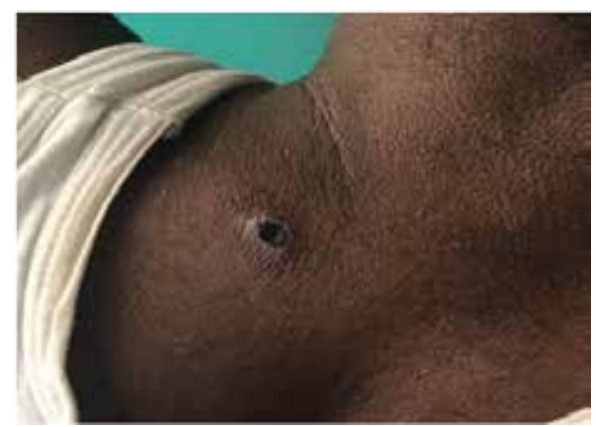

Fig 1: Eschar in the right side of the chest of the patient

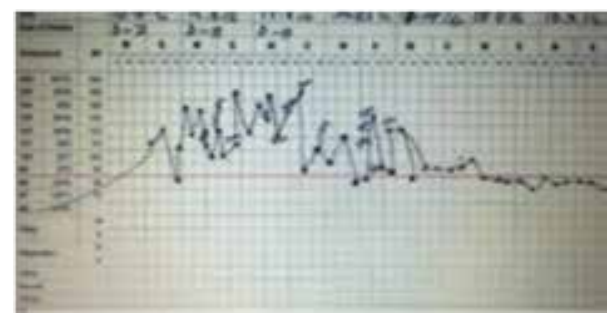

Fig 2: Temperature chart of patient showing high grade fever. After 48 hours of starting doxycycline, fever subsided 


\section{Differential diagnosis}

Our patient presented with meningitis and was empirically treated with ceftriaxone with no improvement. At this time, other less frequent aetiologies were considered.

$M$ tuberculosis, the commonest agent in Bangladesh, can cause meningitis with hypoglycorrhachia. However, the negative tuberculin intradermal reaction, CSF study (Gram stain, Z.N. stain, GeneXpert) and culture did not support this diagnosis.

Viruses can also cause meningitis, namely enteroviruses and arboviruses, especially during summer months, but these were also ruled out by initial presentation of fever for 10 days without any neck rigidity.

\section{Treatment}

Recommended treatment for rickettsial infections is doxycycline $100 \mathrm{mg}$ twice a day for 5-10 days (or at least 3 days following defervescence). ${ }^{1-9}$ Our patient completed 10 days of doxycycline. Cephalosporins and penicillins are ineffective as also observed in this case. ${ }^{9}$

\section{Outcome and follow-up}

The patient evolved favorably with remission of symptoms $48 \mathrm{hr}$ after starting doxycycline and had no sequel.

\section{Discussion}

The atypical presentation of high fever and the paucity of additional symptoms (no rash or eschar), and the fact that Rickettsia meningitis is not a common infectious disease for Bangladesh challenged the initial diagnosis in this case. After non responding to treatment (ceftriaxone) results of CSF study, febrile antigen, and the eschar raised the suspicion of a zoonotic infection and led us to change the antibiotic therapy to doxycycline, with improvement.

Owing to the presence of shared protein and lipopolysaccharide antigens, it is extremely difficult to distinguish closely related agents within the rickettsial spotted fever group by serological methods. ${ }^{10}$ Only successful isolation of the agent or molecular detection in blood or tissue can determine the Rickettsia species. ${ }^{7}$ However, this was not achievable in this case, which might be related to treatment with clarithromycin prior to admission.

Central nervous system involvement, namely Rickettsial encephalitis, is characterized by confusion and obtundation due to increased intracranial pressure and has been associated with a worse prognosis. ${ }^{7}$ Our patient did not show any sign of cerebral parenchymal disease.

In general, Rickettsial meningitis behaves like a viral meningitis but responds only to doxycycline or chloramphenicol instead of symptomatic therapy. ${ }^{11,12}$ Our patient improved with doxycycline. We hypothesize that Rickettsial spp response with doxycycline. The lack of response was due to the short duration of therapy and the erratic central nervous system penetration.

\section{Conclusion}

We conclude that, although Rickettsial meningitis is not common for Bangladesh, Rickettsial infection may present as meningitis and should be included in the differential diagnosis. Definitive diagnosis of Rickettsial infections relies mainly on serological methods that can be limited in an initial phase (due to latency in antibody response), emphasizing the need for a high clinical suspicion. Early treatment should be instituted empirically, since it improves prognosis, and diminishes mortality and sequelae associated with rickettsioses. There are an increasing number of reports regarding emerging Rickettsia spp responsible for incomplete and atypical presentations that should be considered. 


\section{References}

1. Eremeeva ME, Dasch GA. Rickettsial (Spotted \& Typhus Fevers) \& Related Infections (Anaplasmosis \& Erlichiosis). In: Brunette G, editor. CDC Health Information for International Travel 2012 (The Yellow Book). Oxford University Press: England; 2011. p.278-84.

2. Jensenius M, Fournier P, Raoult D. Rickettsioses and the International Traveler. Clin Infect Dis. 2004;39:1493-99.

3. Oteo JA, Portillo A. Tick-Borne Rickettsioses in Europe. Ticks Tick Borne Dis. 2012;3:271-78.

4. de Sousa R, Duque L, Anes M, Pocas J, Torgal J, Bacellar F, et al. Lymphangitis in a Portuguese Patient Infected with Rickettsia sibirica. Emerg Infect Dis. 2008;14:529-30.

5. de Sousa R, Pereira BI, Nazareth C, Cabral S, Ventura C, Crespo P, et al. Rickettsia slovaca Infection in Humans, Portugal. Emerg Infect Dis. 2013;19(10):1627-29.

6. de Sousa R, Nóbrega SD, Bacellar F, Torgal J. Sobrea Realidade da Febre Escaro-Nodular em Portugal. Acta Med Port. 2003;16:429-36. [Article in Spanish]
7. Sousa R, França A, Nóbrega SD, Belo A, Amaro M, Abreu T, et al. Host and Microbial Risk Factors and Pathophysiology of Fatal Rickettsia conorii Infection in Portuguese Patients. J Infect Dis. 2008;198(4): 576-85.

8. Kulkarni A. Childhood Rickettsiosis. Indian J Pediatr. 2011;78:81-87.

9. Eremeeva ME, Dasch GA. Other Rickettsia Species. In: Long SS, Pickering LK, Prober CG, editors. Principles and Practice of Pediatric Infectious Diseases. 3rd ed. Philadelphia, PA: Churcill Livingstone Elsevier; 2008. p. 919-27.

10. Cwikel BJ, Ighbarieh J, Sarov I. Antigenic Polypeptides of Israeli Spotted Fever Isolates Compared with Other Spotted Fever Group Rickettsiae. Ann N Y Acad Sci. 1990;590:381-88.

11. Lindbolm A, Severinson K, Nilsson K. Rickettsia felis Infection in Sweden: Report of Two Cases with Subacute Meningitis and Review of the Literature. Scand J Infect Dis. 2010;42:906-99.

12. Silpapojakul K, Ukkachoke C, Krisanapan S, Silpapojakul K. Rickettsial Meningitis and Encephalitis. Arch Intern Med. 1991;151(9): 1753-57. 